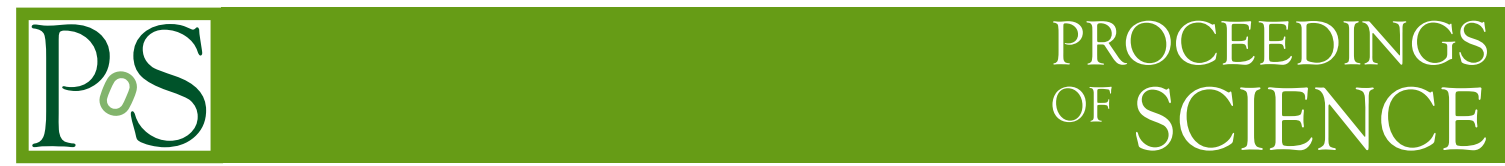

\title{
Charm Semileptonic Decays
}

\section{Arantza Oyanguren ${ }^{* \dagger}$}

Instituto de Física Corpuscular de Valencia (IFIC) (CSIC - U. Valencia)

E-mail: Arantza.Oyanguren@ific.uv.es

Results on charm semileptonic decays from different experiments are reviewed. Measurements of the hadronic contribution involved in these decays are presented and compared with expectations from theoretical models and Lattice QCD results.

The XIth International Conference on Heavy Quarks and Leptons,

June 11-15, 2012

Prague, Czech Republic

*Speaker.

$\dagger$ I would like to thank L. Gibbons and P. Roudeau for the averages. 


\section{Introduction}

Semileptonic decays of charm hadrons provide a way to measure the non perturbative contribution of QCD. The hadronic effects in those decays are expressed in terms of form factors, which are computed using Lattice QCD methods. The validation of Lattice QCD approaches in their calculations is crucial to trust possible New Physics hints in the $b$ quark sector since Lattice results are usually the theoretical inputs entering in the CKM game. Charm semileptonic decays provide in addition a tool to study S-wave systems $\left(J^{P}=0^{+}\right)$interfering with vector mesons in a non-hadronic environment, complementing three body hadronic decay measurements.

Different experiments and techniques are used to measure charm semileptonic decays. At charm threshold colliders, CLEO-c and BES III, tagged and untagged methods are used. In the tagged method one of the D mesons from the $\psi(3770)$ is reconstructed in a hadronic mode and the other $\mathrm{D}$ meson in the semileptonic channel. This procedure gives small background and very good $q^{2}=\left(p_{\bar{\ell}}+p_{v_{\ell}}\right)^{2}$ resolution, where $p_{\bar{\ell}}$ and $p_{v_{\ell}}$ are the four momentum of the lepton and the neutrino, respectively. In the untagged method, used for $D \rightarrow P \bar{\ell} v_{\ell}$, only the lepton and the hadron from the D semileptonic decay are reconstructed, and the neutrino energy is inferred from the rest of the event, giving rise to higher background and poorer resolution but higher statistics. At B-Factories charm semileptonic decays are obtained using different techniques. BaBar partially reconstructs $c \bar{c}$ events from the continuum under the $\Upsilon(4 S)$ resonance, using $D^{*} \rightarrow D \pi$ decays with an electron and a hadron coming from the $\mathrm{D}$ meson. The $\mathrm{D}$ direction and the neutrino energy is obtained using information from the rest of the event, and the background is controlled using large samples of data. At Belle a full reconstruction of $c \bar{c}$ events including hadronization particles is performed. One of the $\mathrm{D}$ is reconstructed into hadrons and the other in the semileptonic channel, being the neutrino the only missing particle in the event. This method leads to high $q^{2}$ resolution but very low efficiency. Measurements have been also performed by previous experiments as the photoproduction experiment FOCUS, where the $D^{*} \rightarrow D \pi$ decay is reconstructed and the D meson decay is selected in the semimuonic channel. Figure 1 shows the signal candidates for $D^{0} \rightarrow$ $K^{-} e^{+} v_{e}$ decays from BaBar [1] using $75 \mathrm{fb}^{-1}$ and for $D^{0} \rightarrow \pi^{-} e^{+} v_{e}$ from preliminary BES III [2] results using $923 \mathrm{pb}^{-1}$.

\section{Pseudoscalar decays: $D \rightarrow(K, \pi, \eta \ldots) \bar{\ell} v_{\ell}$}

The main pseudoscalar modes in charm semileptonic decays are the Cabibbo favoured $D \rightarrow$ $K \bar{\ell} v_{\ell}$ and Cabibbo suppressed $D \rightarrow \pi \bar{\ell} v_{\ell}$ channels. The differential decay width only depends on one form factor ${ }^{1}, f_{+}\left(q^{2}\right)$. Several models and parameterizations are commonly used for $f_{+}\left(q^{2}\right)$. In the Simple Pole and Modified Pole form factors models [3] the $q^{2}$ variation of the form factor is assumed to be governed by a single meson pole or a double pole with an effective pole at higher mass which accounts for other resonant and non-resonant contributions:

$$
f_{+}\left(q^{2}\right)=\frac{f_{+}(0)}{1-\frac{q^{2}}{m_{\text {pole }}^{2}}} \quad \text { and } \quad f_{+}\left(q^{2}\right)=\frac{f_{+}(0)}{\left(1-\frac{q^{2}}{M_{\text {meson }}^{2}}\right)\left(1-\alpha_{\text {pole }} \frac{q^{2}}{M_{\text {meson }}^{2}}\right)} .
$$

Here $f_{+}(0)$ is the normalization of the form factor at $q^{2}=0$. Experiments determine the parameters

\footnotetext{
${ }^{1}$ Neglecting terms proportional to $m_{\ell}$.
} 

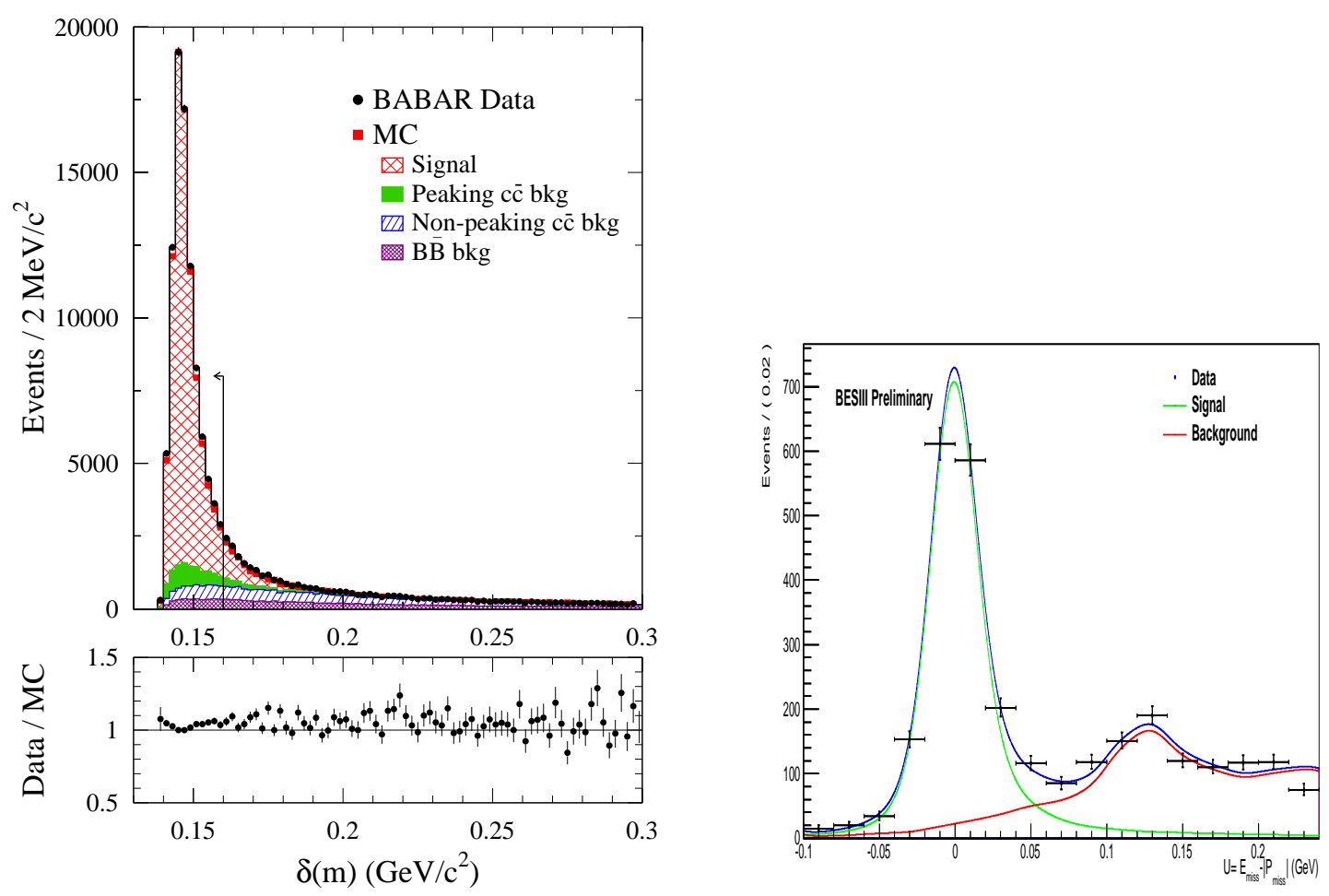

Figure 1: BaBar $D^{0} \rightarrow K^{-} e^{+} v_{e}\left(\delta m=m_{D^{*}}-m_{D^{0}}\right)$ (left) and BES III $D^{0} \rightarrow \pi^{-} e^{+} v_{e}$ (missing energy and momentum difference) (right) signal candidates.

$m_{\text {pole }}$ or $\alpha_{\text {pole }}$, fixing $M_{\text {meson }}$ to the mass of the lowest-lying vector meson contributing to the hadronic current. The normalization of the form factor is also determined at $q^{2}=0$. A general and model independent approach results from a transformation of variables that maps the complex $t=q^{2}$ plane into the open unit disk with center at $t=t_{0}$ [4]. The value of $t_{0}$ depends on the masses of the particles involved. The form factor is expressed as a Taylor expansion in terms of $z\left(t, t_{0}\right) \cdot t_{0}$ is usually defined such that $z=0$ corresponds to the center of the physical $z$-interval.

$$
F(t)=\frac{1}{P(t) \phi\left(t, t_{0}\right)} a_{0}\left(1+\sum_{k=1}^{\infty} r_{k}\left(t_{0}\right) z\left(t, t_{0}\right)^{k}\right) ; \quad|z| \ll 1
$$

$P(t)$ and $\phi\left(t, t_{0}\right)$ are analytical functions; zeroes of $P(t)$ correspond to resonances situated below threshold for open production of states contributing in hadronic currents. The parameters $a_{0}$ and $r_{k}$ are measured by experiments. Recent Lattice QCD calculations of the shape of the form factors $f_{+}^{K}\left(q^{2}\right)$ and $f_{+}^{\pi}\left(q^{2}\right)$ are available [5]. Results of the hadronic form factor parameters obtained by the different experiments are given in Tables 1 and 2 for $m_{\text {pole }}$ and $\alpha_{B K}$, and in Tables 3 and 4 for the $z$ expansion, for the Cabibbo favoured and suppressed channels. Values of $m_{\text {pole }}$ are far from theoretical expectations: the $D_{s}^{*}$ and $D^{*}$ mass for the $D \rightarrow K \bar{\ell} v_{\ell}$ and $D \rightarrow \pi \bar{\ell} v_{\ell}$ channels, respectively. Results on $\alpha_{\text {pole }}$ are below one as expected from the modified form factor model, and can be used to extract information on higher resonance contributions. Experimental results are at present very 
accurate, with a middling agremeent between them. Figures 2 and 3 show the individual results and the combined fit for the $z$ expansion [6]. In Table 5 results on the normalization of the form factor times the corresponding CKM matrix element is given. The experimental accuracy on the form factor normalization is below $1 \%$ for $D \rightarrow K \bar{\ell} v_{\ell}$. There is a good agreement between Lattice QCD and experimental results for both the $D \rightarrow K \bar{\ell} \nu_{\ell}$ and $D \rightarrow \pi \bar{\ell} v_{\ell}$ channels.

Table 1: Results for $m_{\text {pole }}$ and $\alpha_{\mathrm{BK}}$ from various experiments for $D^{0} \rightarrow K^{-} \ell^{+} v_{\ell}$ and $D^{+} \rightarrow K_{S} \ell^{+} v_{\ell}$ decays. The last entry is a lattice QCD prediction.

\begin{tabular}{cccc}
\hline$D \rightarrow K \bar{\ell} v_{\ell}$ Expt. & Ref. & $m_{\text {pole }}\left(\mathrm{GeV} / c^{2}\right)$ & $\alpha_{\mathrm{BK}}$ \\
\hline CLEO III & {$[7]$} & $1.89 \pm 0.05_{-0.03}^{+0.04}$ & $0.36 \pm 0.10_{-0.07}^{+0.03}$ \\
FOCUS & {$[8]$} & $1.93 \pm 0.05 \pm 0.03$ & $0.28 \pm 0.08 \pm 0.07$ \\
BELLE & {$[9]$} & $1.82 \pm 0.04 \pm 0.03$ & $0.52 \pm 0.08 \pm 0.06$ \\
BaBar & {$[1]$} & $1.889 \pm 0.012 \pm 0.015$ & $0.366 \pm 0.023 \pm 0.029$ \\
CLEO-c (tagged) & {$[10]$} & $1.93 \pm 0.02 \pm 0.01$ & $0.30 \pm 0.03 \pm 0.01$ \\
CLEO-c (untagged, $\left.D^{0}\right)$ & {$[11]$} & $1.97 \pm 0.03 \pm 0.01$ & $0.21 \pm 0.05 \pm 0.03$ \\
CLEO-c (untagged, $\left.D^{+}\right)$ & {$[11]$} & $1.96 \pm 0.04 \pm 0.02$ & $0.22 \pm 0.08 \pm 0.03$ \\
BESIII (prel) & {$[2]$} & $1.943 \pm 0.025 \pm 0.003$ & $0.265 \pm 0.045 \pm 0.006$ \\
\hline Fermilab lattice/MILC/HPQCD & {$[12]$} & - & $0.50 \pm 0.04 \pm 0.07$ \\
\hline
\end{tabular}

Table 2: Results for $m_{\text {pole }}$ and $\alpha_{\mathrm{BK}}$ from various experiments for $D^{0} \rightarrow \pi^{-} \ell^{+} v$ and $D^{+} \rightarrow \pi^{0} \ell^{+} v$ decays. The last entry is a lattice QCD prediction.

\begin{tabular}{cccc}
\hline$D \rightarrow \pi \bar{\ell} v_{\ell}$ Expt. & Ref. & $m_{\text {pole }}\left(\mathrm{GeV} / c^{2}\right)$ & $\alpha_{\mathrm{BK}}$ \\
\hline CLEO III & {$[7]$} & $1.86_{-0.06-0.03}^{+0.010+0.07}$ & $0.37_{-0.31}^{+0.20} \pm 0.15$ \\
FOCUS & {$[8]$} & $1.91_{-0.15}^{+0.30} \pm 0.07$ & - \\
BELLE & {$[9]$} & $1.97 \pm 0.08 \pm 0.04$ & $0.10 \pm 0.21 \pm 0.10$ \\
CLEO-c (tagged) & {$[10]$} & $1.91 \pm 0.02 \pm 0.01$ & $0.21 \pm 0.07 \pm 0.02$ \\
CLEO-c (untagged, $\left.D^{0}\right)$ & {$[11]$} & $1.87 \pm 0.03 \pm 0.01$ & $0.21 \pm 0.05 \pm 0.03$ \\
CLEO-c (untagged, $\left.D^{+}\right)$ & {$[11]$} & $1.97 \pm 0.07 \pm 0.02$ & $0.22 \pm 0.08 \pm 0.03$ \\
BESIII (prel) & {$[2]$} & $1.876 \pm 0.023 \pm 0.004$ & $0.315 \pm 0.071 \pm 0.012$ \\
\hline Fermilab lattice/MILC/HPQCD & {$[12]$} & - & $0.44 \pm 0.04 \pm 0.07$ \\
\hline
\end{tabular}

Results on the pseudoscalar decays $D^{+} \rightarrow\left(\eta, \eta^{\prime}\right) e^{+} v_{e}$ have been also provided by the CLEO-c experiment using tagged and untagged methods [22].

3. Vector decays: $D_{(s)} \rightarrow\left(K^{*}, \rho, \phi \ldots\right) \bar{\ell} v_{\ell}$

In case of charm semileptonic decays to vector states the decay rate depends on 5 variables: $q^{2}$, the mass of the vector meson, and three angles $\left(\theta_{V}, \theta_{\ell}\right.$ and $\left.\chi\right)$. Three form factors are gov- 
Table 3: Results for $r_{1}$ and $r_{2}$ from various experiments, for $D \rightarrow K \bar{\ell} v_{\ell}$. The correlation coefficient listed is for the total uncertainties (statistical $\oplus$ systematic) on $r_{1}$ and $r_{2}$.

\begin{tabular}{cccccc}
\hline Expt. $D \rightarrow K \bar{\ell} v_{\ell}$ & mode & Ref. & $r_{1}$ & $r_{2}$ & $\rho$ \\
\hline CLEO III & & {$[7]$} & $0.2_{-3.0}^{+3.6}$ & $-89_{-120}^{+104}$ & -0.99 \\
FOCUS & & {$[8]$} & $-2.54 \pm 0.75$ & $7 \pm 13$ & -0.97 \\
BaBar & & {$[1]$} & $-2.5 \pm 0.2 \pm 0.2$ & $2.5 \pm 6.0 \pm 5.0$ & -0.64 \\
CLEO-c (tagged) & $D^{0} \rightarrow K^{-}$ & {$[10]$} & $-2.65 \pm 0.34 \pm 0.08$ & $13 \pm 9 \pm 1$ & -0.82 \\
CLEO-c (tagged) & $D^{+} \rightarrow \bar{K}^{0}$ & {$[10]$} & $-1.66 \pm 0.44 \pm 0.10$ & $-14 \pm 11 \pm 1$ & -0.82 \\
CLEO-c (untagged) & $D^{0} \rightarrow K^{-}$ & {$[11]$} & $-2.4 \pm 0.4 \pm 0.1$ & $21 \pm 11 \pm 2$ & -0.81 \\
CLEO-c (untagged) & $D^{+} \rightarrow \bar{K}^{0}$ & {$[11]$} & $-2.8 \pm 6 \pm 2$ & $32 \pm 18 \pm 4$ & -0.84 \\
BES III (prel.) & & {$[2]$} & $-2.18 \pm 0.36 \pm 0.05$ & $5 \pm 9 \pm 1$ & \\
\hline \hline Combined [6] & & & $-2.39 \pm 0.17$ & $6.2 \pm 3.8$ & -0.82 \\
\hline
\end{tabular}
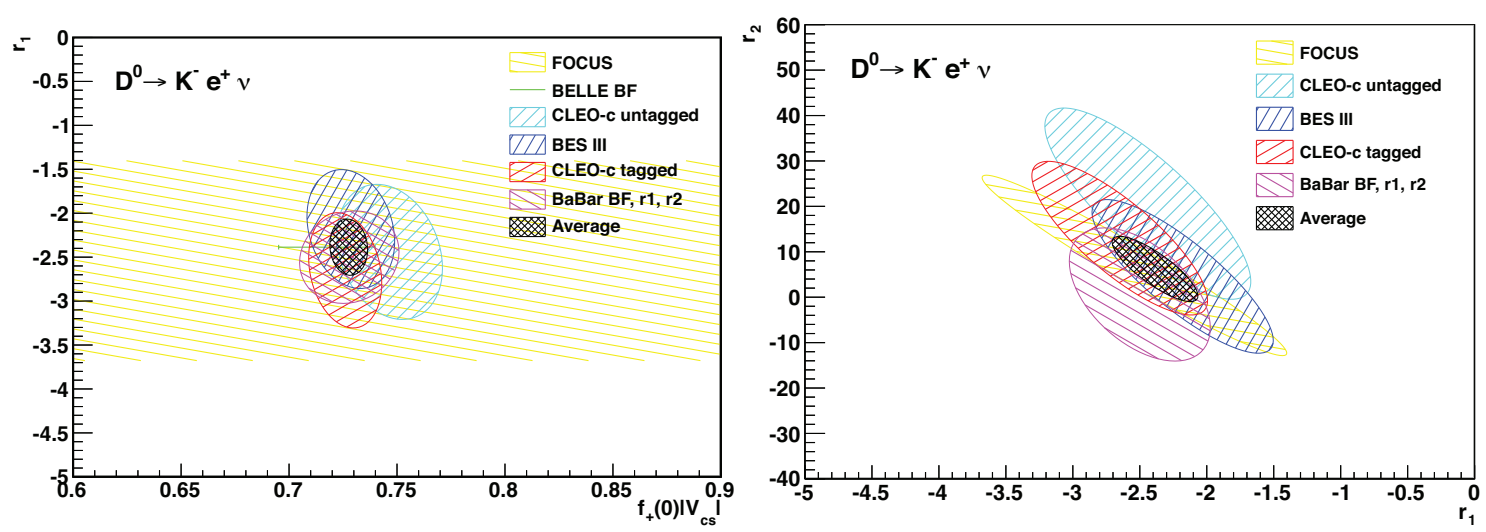

Figure 2: The $D^{0} \rightarrow K^{-} \ell^{+} v_{\ell}$ (left) and $D^{0} \rightarrow \pi^{-} \ell^{+} v_{\ell}$ (right) 68\% C.L. error ellipses from the average fit of the 3-parameter $z$-expansion results from HFAG [6].

Table 4: Results for $r_{1}$ and $r_{2}$ from various experiments, for $D \rightarrow \pi \bar{\ell} v_{\ell}$. The correlation coefficient listed is for the total uncertainties (statistical $\oplus$ systematic) on $r_{1}$ and $r_{2}$.

\begin{tabular}{cccccc}
\hline Expt. $D \rightarrow \pi \bar{\ell} v_{\ell}$ & mode & Ref. & $r_{1}$ & $r_{2}$ & $\rho$ \\
\hline \hline CLEO-c (tagged) & $D^{0} \rightarrow \pi^{+}$ & {$[10]$} & $-2.80 \pm 0.49 \pm 0.04$ & $6 \pm 3 \pm 0$ & -0.94 \\
CLEO-c (tagged) & $D^{+} \rightarrow \pi^{0}$ & {$[10]$} & $-1.37 \pm 0.88 \pm 0.24$ & $-4 \pm 5 \pm 1$ & -0.96 \\
CLEO-c (untagged) & $D^{0} \rightarrow \pi^{+}$ & {$[11]$} & $-2.1 \pm 0.7 \pm 0.3$ & $-1.2 \pm 4.8 \pm 1.7$ & -0.96 \\
CLEO-c (untagged) & $D^{+} \rightarrow \pi^{0}$ & {$[11]$} & $-0.2 \pm 1.5 \pm 0.4$ & $-9.8 \pm 9.1 \pm 2.1$ & -0.97 \\
BES III (prel.) & & {$[2]$} & $-2.73 \pm 0.48 \pm 0.08$ & $4 \pm 3 \pm 1$ & \\
\hline \hline Combined [6] & & & $-2.69 \pm 0.32$ & $4.18 \pm 2.16$ & -0.95 \\
\hline
\end{tabular}



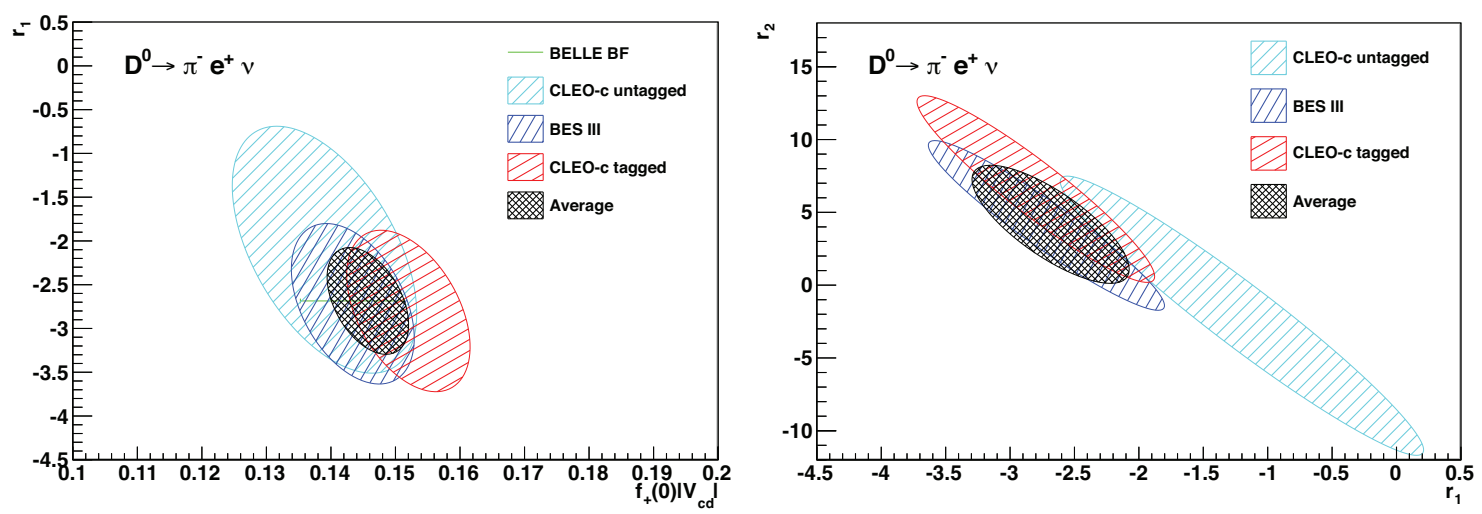

Figure 3: The $D^{0} \rightarrow \pi^{-} \ell^{+} v_{\ell}$ (left) and $D^{0} \rightarrow \pi^{-} \ell^{+} v_{\ell}$ (right) $68 \%$ C.L. error ellipses from the average fit of the 3-parameter z-expansion results from HFAG [6].

\begin{tabular}{cccc}
\hline Experiment & Ref. & $f_{+}^{K}(0)\left|V_{c s}\right|$ & $f_{+}^{\pi}(0)\left|V_{c d}\right|$ \\
\hline \hline E691 (1989) & {$[13]$} & $0.69 \pm 0.05 \pm 0.05$ & \\
CLEO (1991) & {$[14]$} & & \\
CLEOII (1993) & {$[15]$} & $0.76 \pm 0.01 \pm 0.04$ & \\
CLEOII (1995) & {$[16]$} & & $0.163 \pm 0.031 \pm 0.011$ \\
E687 (1995) & {$[17]$} & $0.69 \pm 0.03 \pm 0.03$ & \\
E687 (1996) & {$[18]$} & & $0.160 \pm 0.018 \pm 0.004$ \\
BESII (2004) & {$[19]$} & $0.78 \pm 0.04 \pm 0.03$ & $0.164 \pm 0.032 \pm 0.014$ \\
CLEOIII (2005) $*$ & {$[7]$} & & $0.139_{-0.013}^{+0.011}{ }_{-0.006}^{+0.09}$ \\
FOCUS (2005) & {$[8]$} & & $0.137 \pm 0.008 \pm 0.008$ \\
\hline Belle (2006) $*$ & {$[9]$} & $0.692 \pm 0.007 \pm 0.022$ & $0.140 \pm 0.004 \pm 0.007$ \\
BABAR (2007) * & {$[1]$} & $0.720 \pm 0.007 \pm 0.007$ & \\
CLEO-c (2008)(untagged) * & {$[11]$} & $0.747 \pm 0.009 \pm 0.009$ & $0.139 \pm 0.007 \pm 0.003$ \\
CLEO-c (2009) (tagged) * & {$[10]$} & $0.719 \pm 0.006 \pm 0.005$ & $0.150 \pm 0.004 \pm 0.001$ \\
BESIII (2012)(prel.) * & {$[2]$} & $0.729 \pm 0.008 \pm 0.007$ & $0.144 \pm 0.005 \pm 0.002$ \\
\hline \hline Combined fit $(*)$ & {$[6]$} & $0.728 \pm 0.005$ & $0.146 \pm 0.003$ \\
\hline \hline HPQCD & {$[20][21]$} & $0.727 \pm 0.018$ & $0.150 \pm 0.007$ \\
\hline \hline
\end{tabular}

Table 5: Normalization of the form factors times the CKM matrix element, $f_{+}^{K}(0)\left|V_{c s}\right|$ and $f_{+}^{\pi}(0)\left|V_{c d}\right|$, from different experiments and from Lattice QCD results. Quoted results from Lattice uses values of $V_{c d}=0.2253$ and $V_{c s}=09729$, assuming the unitarity of the CKM matrix. The combined fit includes the results marked with $*[6]$. 
erning these transitions, expressed in terms of two form factor ratios: $R_{2}=A_{2}(0) / A_{1}(0)$ and $R_{V}=V(0) / A_{1}(0)$. The shapes of $A_{1}\left(q^{2}\right), A_{2}\left(q^{2}\right)$ and $V\left(q^{2}\right)$ have been modelled by [23]. In addition, the reconstruction of these decays are sensitive to the $\mathrm{S}$ wave systems through the interference effect with the vector meson. BaBar [24] and CLEO-c [25] have reconstructed $D^{+} \rightarrow K^{-} \pi^{+} \ell^{+} v_{\ell}$ events and measured the form factors in these transtions. BaBar uses $347 \mathrm{fb}^{-1}$ and performed in addtion a detailed study of the contribution and properties of the $K^{*}(892)^{0}$ and higher resonances. $\mathrm{BaBar}$ also determines the S-wave contribution and measures its phase, compatible with expectations from $K \pi$ scattering (see Fig. 4 left). CLEO-c performs a measurement of the $q^{2}$ dependence of the helicity form factors in $D^{+} \rightarrow K^{-} \pi^{+} \ell^{+} v_{\ell}$ decays. Figure 4 (right) shows the comparison of BaBar and CLEO-c measurements of the helicity form factor components $H_{0}\left(q^{2}\right)$ and $h_{0}\left(q^{2}\right)$. Experimental accuracy on form factors is at present much higher than Lattice QCD results. Table 6 lists measurements of $R_{V}$ and $R_{2}$ from several experiments. Most of measurements assume that the $q^{2}$ dependence of hadronic form factors is given by a simple pole.

Table 6: Results for $R_{V}$ and $R_{2}$ from various experiments.

\begin{tabular}{cccc}
\hline Experiment & Ref. & $R_{V}$ & $R_{2}$ \\
\hline$D^{+} \rightarrow \bar{K}^{* 0} l^{+} v$ & & & \\
E691 & {$[26]$} & $2.0 \pm 0.6 \pm 0.3$ & $0.0 \pm 0.5 \pm 0.2$ \\
E653 & {$[27]$} & $2.00 \pm 0.33 \pm 0.16$ & $0.82 \pm 0.22 \pm 0.11$ \\
E687 & {$[28]$} & $1.74 \pm 0.27 \pm 0.28$ & $0.78 \pm 0.18 \pm 0.11$ \\
E791 (e) & {$[29]$} & $1.90 \pm 0.11 \pm 0.09$ & $0.71 \pm 0.08 \pm 0.09$ \\
E791 $(\mu)$ & {$[30]$} & $1.84 \pm 0.11 \pm 0.09$ & $0.75 \pm 0.08 \pm 0.09$ \\
Beatrice & {$[31]$} & $1.45 \pm 0.23 \pm 0.07$ & $1.00 \pm 0.15 \pm 0.03$ \\
FOCUS & {$[32]$} & $1.504 \pm 0.057 \pm 0.039$ & $0.875 \pm 0.049 \pm 0.064$ \\
\hline$D^{0} \rightarrow \bar{K}^{0} \pi^{-} \mu^{+} v$ & & & \\
FOCUS & {$[33]$} & $1.706 \pm 0.677 \pm 0.342$ & $0.912 \pm 0.370 \pm 0.104$ \\
BaBar & {$[24]$} & $1.493 \pm 0.014 \pm 0.021$ & $0.775 \pm 0.011 \pm 0.011$ \\
\hline$D_{s}^{+} \rightarrow \phi e^{+} v$ & & & \\
BaBar & {$[38]$} & $1.636 \pm 0.067 \pm 0.038$ & $0.705 \pm 0.056 \pm 0.029$ \\
\hline$D^{0}, D^{+} \rightarrow \rho e^{+} v$ & & & \\
CLEO & {$[34]$} & $1.40 \pm 0.25 \pm 0.03$ & $0.57 \pm 0.18 \pm 0.06$ \\
\hline
\end{tabular}

CLEO-c has also performed a full reconstruction of the suppresed decay $D \rightarrow \rho e^{+} v_{e}(\rho \rightarrow \pi \pi)$ and measured the form factors ratios [37], which are compatible with the theoretical predictions [23].

Strange charmed meson decays have been measured at BaBar [38] and CLEO-c [39] experiments. BaBar reconstructs $D_{s} \rightarrow K^{+} K^{-} e^{+} v_{e}$ events, and finds that the $K^{+} K^{-}$is primarily coming from the $\phi$ meson decay. A partial reconstruction technique, similar to the one used for the $D^{0} \rightarrow K^{-} e^{+} v_{e}$ and $D^{+} \rightarrow K^{-} \pi^{+} e^{+} v_{e}$ analyses is used. With $214 \mathrm{fb}^{-1} \mathrm{BaBar}$ measures the form factor ratios $R_{V}$ and $R_{2}$ and the simple pole mass $m_{A}$, assumed in the axial vector form factor de- 

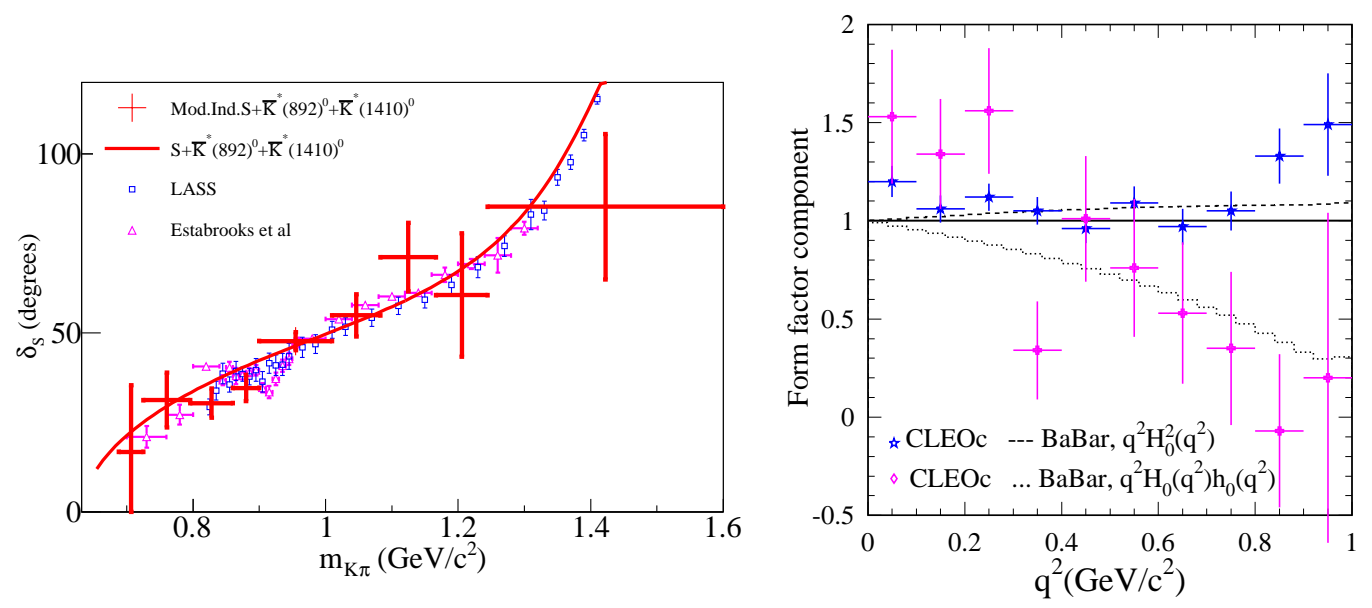

Figure 4: Left: BaBar S-wave measurement (red points) and fitted result (line) compared with $K \pi$ scattering [35] and LASS experiments [36]. Right: Comparison between CLEO-c measurements and BABAR results for the helicity form factors in the $D^{+} \rightarrow K^{-} \pi^{+} \ell^{+} v_{\ell}$ decay channel.

pendence, in the $\phi$ region. The normalization of the form factor is also determined. Results are in agreement with recent Lattice QCD calculations [40] although more precise. A first evidence of a S-wave component is observed (presumably $f_{0} \rightarrow K^{+} K^{-}$), giving a relative small contribution, $\left(0.22_{-0.08}^{+0.12} \pm 0.03\right) \%$ of the total $D_{s} \rightarrow K^{+} K^{-} e^{+} v_{e}$ rate. This result is consistent with the experimental results of a S-wave for the $B_{s} \rightarrow J / \psi K^{+} K^{-}$channel from D0, CDF and LHCb. The CLEO-c experiment with CESR operating at the energy of $4176 \mathrm{MeV}$, reconstructs the semileptonic $D_{s}$ decay into $K^{+} K^{-}$and $\pi^{+} \pi^{-}$. In addition to the $\phi$ meson they observe the $0^{+}$state $f_{0}(980) \rightarrow \pi^{+} \pi^{-}$ and measure its branching fraction. The branching fraction for the $D_{s} \rightarrow \phi e^{+} v_{e}$ decay channel is measured compatible with the $\mathrm{BaBar}$ result, but less precise. Since BaBar cannot reconstruct the final state into pions, both experiments give complementary results of the $f_{0}(980)$ state.

\section{Conclusions}

Measurements of charm semileptonic decays are crucial to validate Lattice QCD calculations of the hadronic effects involved in these decays. The experimental effort in the past years has been remarkable. There are many results from several experiments working at different energy and using different techniques resulting in accurate measurements of the form factors. $\left|V_{c s}\right| f_{+}^{K}(0)$ and $\left|V_{c d}\right| f_{+}^{\pi}(0)$ have reached a precision of $1 \%$ and $2 \%$ respectively. In addition, new data is coming from the BES III experiment, and only partial statistics has been used from the B factories, still having room for improvements. There is also an important activity from the Lattice QCD community, and recent Lattice results are more precise. They are in good agreement with experiments. Previous model parameterizations of the form factors, even if theory dependent, let the experimental results to give a physics interpretation of the hadronic states contribution. Experiments also provide important information on the S-wave systems interfering with vector mesons in charm semileptonic decays. 


\section{References}

[1] B. Aubert et al., Phys. Rev. D 76, 052005 (2007).

[2] BESIII Collaboration, Talk by Chunlei Liu at the CHARM 2012 conference ,http ://www.phys.hawaii.edu/.

[3] D. Becirevic and A. B. Kaidalov, Phys. Lett. B 478, 417 (2000).

[4] T. Becher and R. J. Hill, Phys. Lett. B 633, 61 (2006).

[5] J. A. Bailey et al. [Fermilab and Lattice and MILC Collaborations], PoS LATTICE 2011 (2011) 270 [arXiv:1111.5471 [hep-lat]].

[6] Y. Amhis et al. [Heavy Flavor Averaging Group Collaboration], arXiv:1207.1158 [hep-ex].

[7] G. S. Huang et al., Phys. Rev. Lett. 94, 011802 (2005).

[8] J. M. Link et al., Phys. Lett. B 607, 233 (2005).

[9] L. Widhalm et al., Phys. Rev. Lett. 97, 061804 (2006).

[10] D. Besson et al. [CLEO Collaboration], Phys. Rev. D 80 (2009) 032005 [arXiv:0906.2983 [hep-ex]].

[11] S. Dobbs et al. [CLEO Collaboration], Phys. Rev. D 77 (2008) 112005, [arXiv:0712.1020 [hep-ex]].

[12] C. Aubin et al., Phys. Rev. Lett. 94, 011601 (2005).

[13] J.C. Anjos et al.,(E691 Collaboration), Phys. Rev. Lett. 62, 1587 (1989).

[14] G. Crawford et al., (CLEO Collaboration), Phys. Rev. D44, 3394, (1991).

[15] A. Bean et al., (CLEO Collaboration), Phys. Lett. B317, 647, (1993).

[16] F. Butler et al., (CLEO Collaboration), Phys. Rev. D52, 2656, (1995).

[17] P.L. Frabetti et al., (E687 Collaboration), Phys. Lett. B364, 127, (1995).

[18] P.L. Frabetti et al., (E687 Collaboration), Phys. Lett. B382, 312, (1996).

[19] Ablikim, M.et al., (BES Collaboration), Phys.Lett. B597, 39, (2004).

[20] Na, Heechang and Davies, Christine T.H. and Follana, Eduardo and Koponen, Jonna and Lepage, G. Peter et al., Phys.Rev. D84, 114505 (2011)

[21] Na, Heechang and Davies, Christine T.H. and Follana, Eduardo and Lepage, G. Peter and Shigemitsu, Junko, Phys.Rev. D82, 114506, (2010).

[22] J. Yelton et al. (CLEO Collaboration), Phys. Rev. D 84 (2011) 032001, [arXiv:1011.1195 [hep-ex].

[23] S. Fajfer and J. F. Kamenik, Phys. Rev. D 72Phys. Rev. D 72 (2005) 034029, [hep-ph/0506051].

[24] P. del Amo Sanchezet al., (BABAR Collaboration), Phys. Rev. D83, 072001, (2011).

[25] R. A. Briere et al. [CLEO Collaboration], Phys. Rev. D 81 (2010) 112001, [arXiv:1004.1954 [hep-ex]].

[26] J. C. Anjos et al., Phys. Rev. Lett. 65, 2630 (1990).

[27] K. Kodama et al., Phys. Lett. B 274, 246 (1992).

[28] P. L. Frabetti et al., Phys. Lett. B 307, 262 (1993).

[29] E. M. Aitala et al., Phys. Rev. Lett. 80, 1393 (1998). 
[30] E. M. Aitala et al., Phys. Lett. B 440, 435 (1998).

[31] M. Adamovich et al., Eur. Phys. J. C 6, 35 (1999).

[32] J. M. Link et al., Phys. Lett. B 544, 89 (2002).

[33] J. M. Link et al., Phys. Lett. B 607, 67 (2005).

[34] H. Mahlke, arXiv:hep-ex/0702014 (2007).

[35] P. Estabrooks et al., Nucl. Phys. B133, 490 (1978).

[36] D. Aston et al., Nucl. Phys. B296, 493 (1988).

[37] S. Dobbs et al. [CLEO Collaboration], arXiv:1112.2884 [hep-ex].

[38] B. Aubert et al. [BABAR Collaboration], Phys. Rev. D 78 (2008) 051101 [arXiv:0807.1599 [hep-ex]].

[39] K. M. Ecklund et al. [CLEO Collaboration], Phys. Rev. D 80 (2009) 052009 [arXiv:0907.3201 [hep-ex]].

[40] G. Donald, C. Davies and J. Koponen, arXiv:1111.0254 [hep-lat]. 